\title{
Und Action! Vom Hirn ins Herz bis in die Füße
}

\author{
Michaela Sambanis \& Maik Walter
}

Was ist der Mehrwert eines performativen Unterrichts? Warum wirken die kreativen Ansätze, die den Körper mit in das Lernen einer fremden Sprache einbeziehen? Und wie kann der tertiäre Bildungsbereich im Rahmen eines Embodied Learning von neueren Forschungsarbeiten aus dem Bereich der Neurowissenschaften, der Psychologie und der Didaktik profitieren?

Die beiden Vortragenden stellten ein gemeinsames Projekt vor, das im Frühjahr 2019 bei Cornelsen publiziert wird. In drei Themenfeldern wurden die Bereiche Neurowissenschaften und Performative Didaktik verknüpft und mögliche Synergieeffekte beleuchtet.

In ihrer bewegten Lecture Performance gingen die beiden Vortragenden den eingangs gestellten Fragen nach. Hierzu wurden zunächst ausgewählte Befunde aus der entsprechenden neurowissenschaftlichen Forschung in drei Mini-Inputs kompakt und verständlich referiert. In einem weiteren Schritt wurden diese Wissensbestände mit dramapädagogischen Impulsen für den Fremdsprachenunterricht in Verbindung gesetzt, die gemeinsam ausprobiert wurden.

So wurde beispielsweise die Speicherung von Gedächtnisinhalten erklärt und anschließend von den Teilnehmer*innen der Tagung in eine fantasievollanregende Performance umgesetzt. Durch die Erarbeitung dieser Performance setzten sich alle Teilnehmer*innen bewusst und intensiv mit einer Kernfrage des Fremdsprachenunterrichts auseinander, die zuvor thematisiert wurde: Wie gelangen die lexikalischen Strukturen in den Kopf und vor allem, wie bleiben sie dort in abrufbarer Form erhalten? Die unterschiedliche Speicherung von episodischem oder deklarativem Wissen führte auch zur Wahl von deutlich unterschiedlichen ästhetischen Mitteln in der Performance.

Die aktivierende Ästhetisierung des Wissens ermöglichte den Teilnehmer*innen einen intensiven Austausch.

Mit der Lecture wurde zugleich ein Einblick in die aktuelle performativ ausgerichtete Aus- und Fortbildung von Fremdsprachenlehrer*innen gegeben, denn sie stellte ein von den beiden Vortragenden entwickeltes Workshopformat auf der Basis von Sambanis \& Walter (2019) vor, das bereits mehrfach international getestet wurde. Es eignet sich für alle, die ihren (Fremd) Sprachenunterricht, orientiert an aktuellen Erkenntnissen, performativ ausrichten wollen. 


\section{Bibliografie}

Sambanis, Michael \& Walter, Maik (erscheint 2019): In Motion Theaterimpulse zum Sprachenlernen. Von neuesten Befunden der Neurowissenschaft zu konkreten Unterrichtsimpulsen. Berlin: Cornelsen 